\title{
EXPERIENCIAS DE AGRICULTURA CIRCULAR EN CATALUÑA: DOS CASOS DE ESTUDIO
} Victoria Soldevila $^{\text {a** }}$, Jordi Rosell ${ }^{\mathrm{b}}$, Lourdes Viladomiu ${ }^{\mathrm{b}}$, Karen Hoberg ${ }^{\mathrm{c}}$

${ }^{a}$ Universitat Rovira i Virgili (soldevila@hotmail.com) ${ }^{b}$ UniversitatAutònoma de Barcelona. ${ }^{c}$ Consultora independiente

\section{Resumen}

La economía circular está tomando impulso como estrategia para afrontar la sostenibilidad ambiental. Su implementación en el sector agrario plantea muchos retos entre los que destaca el ámbito en el que encuadrar las actuaciones. En estas líneas nos ocuparemos de dos experiencias de alcance muy diferente.

Palabras clave: Agricultura circular, diversificación agraria, sostenibilidad

\section{Introducción y objetivos}

El concepto de producción circular supone implementar el uso de prácticas y tecnologías que minimizan el uso de los recursos y las emisiones buscando cerrar el ciclo de vida de los productos utilizados o generados en el proceso productivo (De Jesus y Mendonça, 2018; Geissdoerfer et al, 2017; Kirchherr et al. 2017). A pesar de que el concepto de agricultura circular se está popularizando en los estudios agroalimentarios, no existe una definición generalmente aceptada y el concepto se halla sometido a muy diversas interpretaciones.

Una de las cuestiones que plantean los sistemas agroalimentarios es el de la escala óptima para la circularidad, es decir, cuál es la escala a la que debe 'cerrarse el círculo': ¿resulta posible o viable económicamente cerrarlo a nivel micro (explotación agraria)? ¿sería más eficiente plantear la circularidad desde los distintos agentes que configuran la cadena de valor del producto agroalimentario o a nivel de un clúster de empresas ubicadas en un mismo territorio? ¿Qué 'territorio' resulta óptimo para cerrar el círculo: local, comarcal, regional, nacional?

En esta comunicación se presentan dos casos de estudio donde la circularidad es entendida a dos niveles diferentes. En el primero, la circularidad se estudia a nivel de explotación. Cal Rovira es una explotación familiar que ha ido diversificando e integrando su producción. El segundo, a un proyecto institucional de implementar la circularidad territorial a nivel comarcal. Se trata del Programa de Economía Circular promovido en el Berguedà. El análisis de los dos casos de estudio nos ha de permitir analizar la existencia de complementariedades y divergencias en proyectos de economía circular propuestos a escalas diferentes. De dicho análisis se pueden extraerse implicaciones políticas que permitan dilucidar las dificultades y potencialidades para la implementación de iniciativas de agricultura circular.

\section{Metodología}

La literatura sobre economía circular se centra en el sector industrial, la agricultura ha recibido poca atención. Dagevos y de Lauwere (2021) distinguen entre una perspectiva 'adaptativa' (basada en soluciones tecnológicas) y una perspectiva 'alternativa' (basada en cambios profundos en los modelos de producción y consumo). Diversos autores, señalan entre las limitaciones de la literatura académica sobre la agricultura circular, la falta de trabajos empíricos especialmente a nivel micro (Zucchella y Previtali, 2019; Zhu et al. 2019). Además, los escasos trabajos empíricos y estudios de casos de explotaciones agrarias se centran en un concepto de circularidad basado en desarrollos tecnológicos (por ejemplo, plantas de biogás en explotaciones ganaderas) y no en una circularidad basada en la diversificación y actividades complementarias en la misma explotación, como el modelo que presentamos en este artículo. En este sentido, destacamos la originalidad de nuestra aportación. Tampoco existen estudios de caso donde se discuta las posibilidades de la complementariedad a diferentes escalas.

La metodología utilizada es la de casos de estudio, utilizando técnicas de recogida de datos y realización de entrevistas semi estructuradas a los gestores de la explotación y a la Administración comarcal.

\section{Resultados}

\subsection{Circularidad en el ámbito de una explotación agraria}

Tancant Cercles/Cal Rovira (Cerrando círculos/Cal Rovira) integra un amplio abanico de empresas y actividades agroalimentarias cuya base es una familia de agricultores y tres masías en propiedad. Cuando la familia Rovira optó por el nombre comercial de Tancant Cercles lo hizo desde una perspectiva sectorial. La producción porcina se situaba en el centro del círculo y cerrarlo era el mecanismo para valorizar un producto diferenciado y llegar hasta el consumidor final. Pero un análisis detallado de la estrategia de la explotación permite apreciar el encaje de ésta con los elementos que hoy se persiguen en las propuestas de economía circular.

La estrategia de Cal Rovira parte de del carácter agro-pecuario-forestal propio de las masías tradicionales, con un amplio abanico de activos (bosques, tierras cultivables, pastos, edificaciones,) y producciones 
(huerta, cereales, forrajes, aves, porcino, bovino...) junto con el capital humano que aportan los miembros de la familia.

En las últimas dos décadas la familia ha sabido re-inventar la masía adaptándola a las condiciones presentes para hacerla viable pero sin perder el carácter unitario de la explotación. Para ello a la vez que se ha hecho un esfuerzo, no siempre recompensado económicamente, para conservar actividades tradicionales (cuidado del bosque, bovino en pastos,) se han creado y modernizado actividades (obrador cárnico, molino de colza, turismo rural, restaurantes gastronómicos) y desarrollado nuevos circuitos de comercialización (circuitos cortos, venta online, restaurantes propios, etc.). Los activos de la explotación continúan siendo la base de las actividades del grupo, la diversificación y la circularidad se ha profundizado (los cultivos y el molino les permite producir buena parte de los alimentos para su ganado, los restaurantes ofrecen salida a una parte de su producción de huerta y cárnicos).

La circularidad comporta mantener algunas actividades que serían más rentables si se externalizaran o si se sustituyeran por inputs adquiridos en el mercado. Sin embargo, esta estrategia permite la mayor valorización de los inputs disponibles en la explotación, minimizando residuos. Así, por ejemplo, la ganadería de la finca proporciona estiércol que permite la reducción del uso de fertilizantes químicos, les permite limpiar el bosque y disminuir el riesgo de incendio; la ganadería, les garantiza unos niveles de imagen y calidad para los productos que elaboran. La circularidad se convierte en un activo intangible de la explotación que aporta identidad al grupo y a los bienes y servicios que comercializa bajo el lema de Tancant Cercles.

Conceptualmente, la estrategia de circularidad de Cal Rovira puede considerase como una alternativa a la estrategia de modernización, especialización y aprovechamiento de las economías de escala. Mantiene la diversificación propia de las masías, aprovecha las economías de alcance o gama, amplia círculos al tiempo que hace una apuesta fuerte por las tecnologías ajustándolas a la circularidad. Todo se valoriza en el propio entramado del grupo empresarial. Este modelo elude las dificultades y la incertidumbre vinculada de comercialización de subproductos y residuos que conllevan otros modelos de economía circular.

Si analizamos la experiencia de Cal Rovira desde los siete elementos de circularidad propuestos en la Estrategia Española de Economía Circular (MITECO, 2020), se observa que la empresa destaca en los objetivos de conseguir una mayor eficiencia en el uso de insumos en la producción impulsando el uso de materiales reciclados y materias primas secundarias (un 70-80\% de la alimentación del ganado proviene de la propia explotación) y de la reducción de los residuos no valorados, evitando la generación de residuos, lo que comporta en consecuencia la reducción del desperdicio alimentos. Estos tres principios han estado siempre en la base de la actuación de las masías, de forma que parecen cumplirse de forma natural como consecuencia del propio modelo de producción y explotación de las fincas.

Igualmente, al realizar en paralelo actividades agrícolas, ganaderas y forestales estas permiten reducir el uso de productos químicos y mejorar la conservación de los suelos y la biodiversidad. Sin embargo, la explotación no ha realizado el paso a la agricultura ecológica a pesar de que lo están estudiando desde hace unos años. Tampoco se ha realizado ninguna actuación específica encaminada a mejorar la eficiencia en el uso del agua, ni en la minimización de las emisiones de gases, pero sí han ido invirtiendo en eficiencia energética.

Aunque Cal Rovira se ha beneficiado de ayudas públicas, la estrategia de diversificación y de circularidad encuentra dificultades para ajustarse a los marcos regulatorios. Las normas están pensadas para explotaciones especializadas, cuyas actividades afectan a un número muy limitado de acciones y trámites. Asimismo, los regímenes de ayuda entran en contradicción cuando una empresa agraria amplía su ámbito de actuación a actividades industriales y de servicio. La tramitación administrativa y la burocracia exigen una complejidad muy costosa en los esquemas jurídicos de las empresas rurales diversificadas y con actividades multifuncionales, lo que actúa como un freno en la implementación de la agricultura circular a nivel micro.

\subsection{La circularidad a nivel de territorio}

La comarca del Berguedà se sitúa en la zona central de Catalunya. Un $86 \%$ del territorio son bosques y en la producción agropecuaria tienen protagonismo las explotaciones ganaderas (porcino y bovino). Los cultivos se limitan a forrajes y cereales. La comarca fue pionera en Catalunya en el lanzamiento de una propuesta comarcal de Economía Circular. El proyecto 'Economía Circular del Berguedà' se inicia en 2018, impulsado por la Agencia de Desarrollo del Berguedà y el Grupo Leader y se concretó en dos acciones. La primera, un análisis para detectar oportunidades para fomentar la economía circular en la comarca en el que se apreció que el sector agrario, ganadero y alimentario presentaba muchas oportunidades de ahorro y mejora ambiental en el ámbito de la circularidad. Mas adelante se realizó un nuevo estudio en donde a través de visitas a explotaciones agropecuarias, se identificaron una serie de actores que integran el 'ecosistema' comarcal y se propone conectar el sector agroalimentario, el de energía y la gestión de los residuos orgánicos. La segunda actuación fue el desarrollo de un toolkit específico (Encercla) para valorar 
el grado de circularidad en las empresas solicitantes de ayudas e inspirado en el desarrollado por la Fundación Ellen Macarthur. En este sentido, se trata de una acción pionera en el mundo rural catalán.

El proyecto ofrecía asesoramiento a empresas y explotaciones y promoción de iniciativas conjuntas para mejorar su eficiencia energética y ambiental. Las oportunidades/necesidades detectadas se concentraron en 4 líneas de actuación: aumentar la eficiencia en la producción (especialmente en granjas porcinas), comercializar en circuitos cortos, compartir recursos (personal, maquinaria), y reducir residuos y aprovechamiento de subproductos. Sin embargo, la única acción emprendida hasta el momento ha sido la gestión de los residuos plásticos de las explotaciones.

\section{Conclusiones}

El análisis de los dos casos de estudio permite apreciar que bajo la noción de circularidad se esconden propuestas, visiones y realidades muy diversas. De un lado, se aprecia que a nivel micro los paradigmas apropiados para la agricultura circular son los modelos de explotación agraria diversificados, basados en economías de alcance en donde las actividades agrarias, ganaderas y forestales se entremezclan y donde se consigue mayor valor añadido incorporando la transformación y suministrando servicios. El caso de la explotación Cal Rovira, es el ejemplo y el modelo de la masia ha sido el origen que ha facilitado la estrategia. Pero su éxito exige una dimensión importante en recursos físicos, capital y humanos. Además, el marco jurídico- normativo no se adecua a este tipo de empresas. No existe un status de empresa rural diversificada que pudiera facilitar el ajuste institucional al modelo de circularidad a nivel micro.

De otro lado, se aprecia que la circularidad a nivel territorial choca con una multitud de sectores y situaciones y que resulta difícil entremezclarlos con una acción institucional desde las Administraciones locales. Sin embargo, cuando en el Berguedà se orientaron a acciones específicas que permitieran una mejor utilización de los residuos de materia orgánica, que se producen en gran cantidad en el sector agroalimentario, se han detectado oportunidades de hacer la transición hacia un modelo productivo más sostenible y eficaz. Es por el momento demasiado pronto para poder determinar si los resultados serán significativos, pero tienen la impresión que para obtener resultados y credibilidad se deberá actuar con acciones muy bien definidas y de alcance limitado, al tiempo que estas deben ser parte de políticas y actuaciones de mayor envergadura impulsadas a nivel autonómico, estatal y europeo. La experiencia del Berguedà indica que una aproximación de la circularidad desde una escala "clúster"- sector agroalimentario- puede ser más apropiada que la escala territorial comarcal como unidad de acción. Asimismo, la experiencia del programa Leader, con la implementación del toolkit Encercla por el momento ha actuado como un instrumento de sensibilización y de abrir inquietudes a los solicitantes de ayudas.

Por último, del estudio de casos se aprecia que las estrategias de incorporación de soluciones tecnológicas puntuales que favorezcan la circularidad (estrategias "adaptativas"), resultan mucho más fáciles de desarrollar e incentivar desde las instituciones, mientras que las estrategias "alternativas" requieren un cambio en profundidad de los modelos empresariales. Para explotaciones como Cal Rovira la implementación de un modelo alternativo choca con un marco institucional- legislativo que no se adecua a los requerimientos de ir cerrando círculos a nivel micro.

\section{Bibliografía}

Dagevos, H., \& de Lauwere, C. (2021). "Circular Business Models and Circular Agriculture: Perceptions and Practicesof Dutch Farmers". Sustainability, 13(3), 1282.

De Jesus, A., \&Mendonça, S. (2018)."'Lost in transition? Drivers and barriers in the eco-innovation road to the circular economy". Ecological economics, 145, 75-89.

Geissdoerfer, M., Savaget, P., Bocken, N., \&Hultink, E. (2017). "The circulareconomy-A new sustainability paradigm?".Journal of Cleaner Production,143, 757-768

Kirchherr, J., Reike, D., \&Hekkert, M. (2017). "Conceptualizing the circular economy: An analysis of 114 definitions". Resources, conservation and recycling, 127, 221-232.

\section{MITECO (2020). España Circular 2030, Madrid}

Zucchella, A., \&Previtali, P. (2019). "Circular business models for sustainable development: A "waste is food" restorative ecosystem". Business Strategy and the Environment, 28(2), 274-285

Zhu, Q., Jia, R., \& Lin, X. (2019). "Building sustainable circular agriculture in China: economic viability and entrepreneurship". Management decision. 